\title{
On the Calculation of the Approximate Values of Stokes' Multipliers
}

\author{
By \\ Mitsuhiko KoHno
}

\section{Introduction}

The study of the system of linear ordinary differential equation

$$
t \frac{d X}{d t}=(A+t B) X
$$

was initiated by G. D. Birkhoff [1] and was continued by many authors [2], [3], [4], [5]. Always the aim of these authors have been the investigation of "the solutions in the large".

The system has two singular points in the entire complex $t$ plane : one is a regular singular point at $t=0$ and the other is an irregular singular point of rank 1 at $t=\infty$. Locally, in the neighbourhood of the regular singular point $t=0$, we have a fundamental set of solutions in convergent form

$$
X_{j}(t)=t^{\rho} \sum_{m=0}^{\infty} G_{j}(m) t^{m} \quad(j=1,2, \cdots, n) .
$$

and near the irregular singular point at infinity, we have a set of formal solutions

$$
X^{k}(t) \sim e^{\lambda_{k} t} t^{a_{k k}} \sum_{s=0}^{\infty} H^{k}(s) t^{-s} \quad(k=1,2, \cdots, n),
$$

and if we restrict ourselves in an arbitrary but sufficiently small sectorial neighbourhood $S$ of this irregular singular point, we have a fundamental set of solutions with the asymptotic behavior as follows,

$$
X_{S}^{k}(t) \cong X^{k}(t) \quad(t \in S)
$$


By the theory of linear differential equations, these two sets of solutions must be connected linearly

$$
X_{j}(t)=\sum_{k=1}^{n} T_{j}^{k}(S) X_{S}^{k}(t) \quad(j=1,2, \cdots, n) .
$$

The constants $T_{j}^{k}(S)$ are called Stokes' multipliers, and because of their discontinuous dependence on the choice of the sectorial neighbourhood $S$, exact evaluations of these Stokes' multipliers have been a difficult and important problem, called a two point connection problem, both theoretically and practically.

Theoretically, an ingenious method of determining Stokes' multipliers was proposed by $K$. Okubo in the paper [4].

Here, we shall briefly explain that method. We write the formal solutions (3) in the form

$$
X^{k}(t) \sim x^{k}(t) \sum_{s=0}^{\infty} H^{k}(s) t^{-s}
$$

with scalar quantities

$$
x^{k}(t)=e^{\lambda_{k} t} t^{a_{k k}} .
$$

Now, we try to construct the scalar entire functions $x_{j}^{k}(t)$ with the asymptotic behavior

$$
t^{\rho_{j}} x_{j}^{k}(t) \cong x^{k}(t)
$$

near infinity. In fact, if we define $x_{j}^{k}(t)$ as holomorphic solutions of the following differential equations that we call the "fundamental differential equations",

$$
t \frac{d x_{j}^{k}(t)}{d t}=\left(\lambda_{k} t+a_{k k}-\rho_{j}\right) x_{j}^{k}(t)+1 \quad(j, k=1,2, \cdots, n) .
$$

and write these solutions in the form

$$
\left\{\begin{aligned}
x_{j}^{k}(t) & =\sum_{m=0}^{\infty} g_{j}^{k}(m) t^{m} \\
g_{j}^{k}(m) & =\frac{\lambda_{k}^{m} \Gamma\left(\rho_{j}-a_{k k}\right)}{\Gamma\left(m+\rho_{j}-a_{k k}+1\right)},
\end{aligned}\right.
$$

then we have the relations between $x_{j}^{k}(t)$ and $x^{k}(t)$ in a certain sectorial neighbourhood of infinity as follows, 


$$
x_{j}^{k}(t) \cong \lambda_{k}^{a_{k k}-\rho_{j}} \Gamma\left(\rho_{j}-a_{k k}\right) e^{\lambda_{k} t} t^{a_{k k}-\rho_{j}}
$$

i.e.,

$$
t^{\rho_{j}} x_{j}^{k}(t) \cong \lambda_{k}^{a_{k k}-\rho_{j}} \Gamma\left(\rho_{j}-a_{k k}\right) x^{k}(t) .
$$

Here, if we substitute $t^{\rho_{j}} x_{j}^{k}(t) / \lambda_{k}^{a_{k k}{ }^{-\rho_{j}}} \Gamma\left(\rho_{j}-a_{k k}\right)$ for $x^{k}(t)$ in the formal solutions (3) and calculate formally, we have

$$
\begin{gathered}
X^{k}(t) \cong\left[t^{\rho_{j}} x_{j}^{k}(t) / \lambda_{k}^{a_{k k}-\rho_{j}} \Gamma\left(\rho_{j}-a_{k k}\right)\right]\left[\sum_{s=0}^{\infty} H^{k}(s) t^{-s}\right] \\
=\frac{\lambda_{k}^{\rho_{j}-a_{k k}}}{\Gamma\left(\rho_{j}-a_{k k}\right)} t^{\rho_{j}}\left[\sum_{m=0}^{\infty} g_{j}^{k}(m) t^{m}\right]\left[\sum_{s=0}^{\infty} H^{k}(s) t^{-s}\right] \\
=\frac{\lambda_{k}^{\rho_{j}-a_{k k}}}{\Gamma\left(\rho_{j}-a_{k k}\right)} t^{\rho_{j}} \sum_{m=-\infty}^{\infty}\left[\sum_{s=0}^{\infty} H^{k}(s) g_{j}^{k}(m+s)\right] t^{m} .
\end{gathered}
$$

We denote the coefficients of $t^{m}$ in these power series by $F_{j}^{k}(m)$, i.e.,

$$
F_{j}^{k}(m)=\sum_{s=0}^{\infty} H^{k}(s) g_{j}^{k}(m+s) .
$$

Then, if we could prove the convergence of the factorial series of $s$ in the right hand side of (10), we could expect to obtain the relations between the coefficients $G_{j}(m)$ of convergent power series solutions (2) and the coefficients $F_{j}^{k}(m)$ from the linear combination (5) and the formulas (4), (9).

$$
\begin{aligned}
X_{j}(t) & =t^{\rho_{j}} \sum_{n=0}^{\infty} G_{j}(m) t^{m} \\
& =\sum_{k=1}^{n} T_{j}^{k}(S) X_{S}^{k}(t) \\
& \simeq \sum_{k=1}^{n} T_{j}^{k}(S)\left[\frac{\lambda_{k}^{\rho_{j}-a_{k k}}}{\Gamma\left(\rho_{j}-a_{k k}\right)} t^{\rho_{j}} \sum_{m=-\infty}^{\infty} F_{j}^{k}(m) t^{m}\right] \\
& =t^{\rho_{j}} \sum_{n=-\infty}^{\infty}\left[\sum_{k=1}^{n} T_{j}^{k}(S) \frac{\lambda_{k}^{\rho_{j}-a_{k k}}}{\Gamma\left(\rho_{j}-a_{k k}\right)} F_{j}^{k}(m)\right] t^{m} .
\end{aligned}
$$

Therefrom, we obtain for non-negative integers

$$
G_{j}(m)=\sum_{k=1}^{n} \bar{T}_{j}^{k}(S) F_{j}^{k}(m)
$$

where we put

$$
\bar{T}_{j}^{k}(S)=T_{j}^{k}(S) \frac{\lambda_{k}^{\rho_{j}-a_{k k}}}{\Gamma\left(\rho_{j}-a_{k k}\right)} \quad(j, k=1,2, \cdots, n) .
$$


From the relations (12), we will be able to determine Stokes' multipliers $T_{j}^{k}(S)$ theoretically by only solving the linear equation for a certain non-negative integer $m_{0}$ and then, from the initial condition of the coefficients $G_{j}(m)$ which satisfy a system of difference equations, all the coefficients of negative powers of $t$ in the right hand side of (11) vanish.

However, in general, we cannot expect to get the values of $F_{j}^{k}(m)$ explicitly, because $F_{j}^{k}(m)$ are represented by the factorial series including an infinite number of the coefficients $H^{k}(s)$ of formal solutions.

The convergence of the factorial series (10) was proved by investigating the location of singular points of certain auxiliary systems of differential equations under the pentagonal condition in the papers [4], [5]; and all, or only some part of Stokes' multipliers can be calculated in case of two, or more than three dimensional system of differential equations respectively.

The author proved the convergence by the simple method of norm estimation in the paper [6].

The object of the present paper is to show that our method, which was used to relax the pentagonal condition, is useful not only theoretically, but practically in the approximate evaluation of Stokes' multipliers with sharp error estimates. This will be demonstrated by computing Stokes' multipliers for Airy's equation by our method and by comparing with the known exact values of them as a special example of a general system of ordinary differential equations of the type (1).

In section 5 , together with a new result for an explicit value of the determinant for the system (12), we will show how our method works for a general system of higher dimensions.

The author would like to express his sincere thanks to Professor M. Hukuhara for his constant counsel and warm encouragement and to Professor K. Okubo for his kind advice. 


\section{Exact Values of Stokes' Multipliers \\ (Method of contour integration)}

Ordinarily, to obtain exact values of Stokes' multipliers, integral representation of solutions of a given ordinary differential equation is used and the saddle point method is applied to those integrals to derive asymptotic behaviors.

In this section, we shall explain briefly the two point connection problem for Airy's equation and compute the exact values of Stokes' multipliers by this method.

Airy's equation

$$
\frac{d^{2} x(t)}{d t^{2}}=t x(t)
$$

has one set of holomorphic solutions in the neighbourhood of a regular point $t=0$ as follows,

$$
\left\{\begin{array}{l}
x_{0}(t)=\sum_{k=0}^{\infty} d_{k} t^{3 k} \\
x_{1}(t)=t \sum_{k=0}^{\infty} \bar{d}_{k} t^{t_{k}}
\end{array}\right.
$$

where

$$
\begin{cases}d_{9}^{k}=\frac{\Gamma\left(\frac{2}{3}\right)}{9^{k} \Gamma(k+1) \Gamma\left(k+\frac{2}{3}\right)}, & d_{0}=1 \\ \bar{d}_{9}^{k}=\frac{\Gamma\left(\frac{4}{3}\right)}{9^{k} \Gamma(k+1) \Gamma\left(k+\frac{4}{3}\right)}, & \bar{d}_{0}=1\end{cases}
$$

and another set of solutions which has the asymptotic behavior in a certain sectorial neighbourhood of $t=\infty$ as follows,

$$
\left\{\begin{array}{l}
x^{1}(t) \cong e^{(2 / 3) t^{(3 / 2)}} t^{-1 / 4}\left[\sum_{k=0}^{\infty} c_{k} t^{-(3 / 2) k}\right] \\
x^{2}(t) \cong e^{-(2 / 3) t^{(3 / 2)}} t^{-1 / 4}\left[\sum_{k=0}^{\infty} \bar{c}_{k} t^{-(3 / 2) k}\right]
\end{array}\right.
$$

where $c_{0}=1, \bar{c}_{0}=1$ and 


$$
c_{k}=(-1)^{k} \bar{c}_{k}=\frac{\Gamma\left(k+\frac{1}{6}\right) \Gamma\left(k+\frac{5}{6}\right)}{\left(\frac{4}{3}\right)^{k} k ! \Gamma\left(\frac{1}{6}\right) \Gamma\left(\frac{5}{6}\right)}, k \geqq 1 .
$$

On the other hand, we have the following integral representations of solutions of Laplace type

$$
x_{j k}(t)=\int_{\Gamma_{j}-\Gamma_{k}} e^{\left(t s-\left(s^{3} / 3\right)\right)} d s, \quad(j, k=0,1,2 ; j \neq k)
$$

where $\Gamma_{j}$ denotes the path of integration as follows,

$$
\Gamma_{j}: s=r e^{(2 \pi i / 3) j} \quad(0 \leqq r<\infty) \quad(j=0,1,2) .
$$

Then, from these integral representations we obtain the power series expansions of solutions at $t=0$,

$$
\begin{aligned}
& x_{j k}(t) \\
& =\left(e^{(2 \pi i / 3) j} \cdot 3^{-2 / 3}\right) \sum_{m=0}^{\infty} \frac{\Gamma\left(\frac{m+1}{3}\right)}{\Gamma(m+1)}\left(t e^{(2 \pi i / 3) j} \cdot 3^{1 / 3}\right)^{m} \\
& \quad-\left(e^{(2 \pi i / 3) k} \cdot 3^{-2 / 3}\right) \sum_{m=0}^{\infty} \frac{\Gamma\left(\frac{m+1}{3}\right)}{\Gamma(m+1)}\left(t e^{(2 \pi i / 3) k} \cdot 3^{1 / 3}\right)^{m}, \\
& \quad(j, k=0,1,2 ; j \neq k)
\end{aligned}
$$

and we take two solutions $x_{01}(t), x_{12}(t)$ as the fundamental set of solutions of Airy's equation (13).

Hence, the solutions $x_{0}(t)$ and $x_{1}(t)$ can be represented by the linear combination of the above fundamental solutions $x_{01}(t)$ and $x_{12}(t)$ as follows,

$$
\left\{\begin{array}{l}
x_{0}(t)=A_{0} x_{01}(t)+B_{0} x_{12}(t) \\
x_{1}(t)=A_{1} x_{01}(t)+B_{1} x_{12}(t) .
\end{array}\right.
$$

The constants $A_{0}, B_{0}, A_{1}$ and $B_{1}$ can be calculated by the values and the values of the first derivatives of solutions at $t=0$ and we obtain

$$
\begin{cases}A_{0}= & \frac{\Gamma\left(\frac{2}{3}\right) 3^{-1 / 3} \sqrt{3} i}{2 \pi i}, \quad B_{0}=\frac{\Gamma\left(\frac{2}{3}\right) 3^{-1 / 3}\left(1-e^{4 \pi i / 3}\right)}{2 \pi i} \\ A_{1} & =\frac{\Gamma\left(\frac{1}{3}\right) 3^{-2 / 3} \sqrt{3} i}{2 \pi i}, \quad B_{1}=\frac{\Gamma\left(\frac{1}{3}\right) 3^{-2 / 3}\left(e^{2 \pi i / 3}-1\right)}{2 \pi i} .\end{cases}
$$


Now we shall investigate the behaviors of the solutions $x_{0}(t)$ and $x_{1}(t)$ in the neighbourhood of infinity. For that purpose, we only seek the asymptotic behaviors of $x_{01}(t)$ and $x_{12}(t)$ in a certain sectorial neighbourhood of $t=\infty$ because of the above relations (21).

In order to obtain the asymptotic forms of $x_{01}(t)$ and $x_{12}(t)$, we apply the saddle point method to the integrals of the extended Laplace type (18), and we have

$$
\left\{\begin{aligned}
& x_{01}(t) \cong \sqrt{\pi} e^{(2 / 3) t^{(3 / 2)}} t^{-1 / 4} \sum_{k=0}^{\infty} \frac{\Gamma\left(k+\frac{1}{6}\right) \Gamma\left(k+\frac{5}{6}\right)}{\left(\frac{4}{3}\right)^{k} \Gamma(k+1) \Gamma\left(\frac{1}{6}\right) \Gamma\left(\frac{5}{6}\right)} t^{-(3 / 2) k} \\
&=\sqrt{\pi} x^{1}(t)\left(|\arg t|<\frac{\pi}{3}\right) \\
& x_{12}(t) \cong \sqrt{\pi} i e^{-(2 / 3) t^{(3 / 2)}} t^{-1 / 4} \sum_{k=0}^{\infty} \frac{(-1)^{k} \Gamma\left(k+\frac{1}{6}\right) \Gamma\left(k+\frac{5}{6}\right)}{\left(\frac{4}{3}\right)^{k} \Gamma(k+1) \Gamma\left(\frac{1}{6}\right) \Gamma\left(\frac{5}{6}\right)^{k}} t^{-(3 / 2) k} \\
&=\sqrt{\pi} i x^{2}(t)\left(|\arg t|<\frac{\pi}{3}\right) .
\end{aligned}\right.
$$

From (21), (22) and (23), we obtain the following connection formulas of two sets of fundamental solutions of Airy's equation in the sector $|\arg t|<\frac{\pi}{3}$.

$$
\left\{\begin{array}{l}
x_{0}(t)=\frac{\Gamma\left(\frac{2}{3}\right) 3^{1 / 6}}{2 \sqrt{\pi}} x^{1}(t)+\frac{\Gamma\left(\frac{2}{3}\right) 3^{1 / 6} e^{(\pi / 6) i}}{2 \sqrt{\pi}} x^{2}(t) \\
x_{1}(t)=\frac{\Gamma\left(\frac{1}{3}\right) 3^{-1 / 6}}{2 \sqrt{\pi}} x^{1}(t)-\frac{\Gamma\left(\frac{1}{3}\right) 3^{-1 / 6} e^{-(\pi / 6) i}}{2 \sqrt{\pi}} x^{2}(t) .
\end{array}\right.
$$

\section{Approximate Values of Stokes' Multipliers}

(Estimation method)

We shall calculate the values of Stokes' multipliers approxi-mately by our method of norm estimation in this section.

For that purpose, we first have to reduce the single Airy's equation (13) to a system of differential equation of the type (1).

Here we change the variable by 


$$
\bar{t}=\frac{2}{3} t^{3 / 2}
$$

and denote $\bar{t}$ again by $t$. Starting from the vector

$$
X(t)=\left(\begin{array}{l}
x_{1}(t) \\
x_{2}(t)
\end{array}\right)
$$

with $x_{1}(t)=x(t), x_{2}(t)=x^{\prime}(t)$, we perform the constant linear transformation

$$
X=P \bar{X}
$$

where the constant matrix $P$ is

$$
P=\left(\begin{array}{rr}
1 & 1 \\
1 & -1
\end{array}\right)
$$

Then, we obtain the desired system of differential equation as follows,

$$
t \frac{d \bar{X}}{d t}=\left\{\left(\begin{array}{rr}
-\frac{1}{6} & \frac{1}{6} \\
\frac{1}{6} & -\frac{1}{6}
\end{array}\right)+t\left(\begin{array}{rr}
1 & 0 \\
0 & -1
\end{array}\right)\right\} \bar{X}
$$

Here the eigenvalues of the first matrix $A$ on the right are $\rho_{1}=0, \rho_{2}=-\frac{1}{3}$ and the eigenvalues of the second matrix $B$ on the right are $\lambda_{1}=1, \lambda_{2}=-1$.

The system of differential equation (28) has the following convergent power series solutions in the neighbourhood of $t=0$,

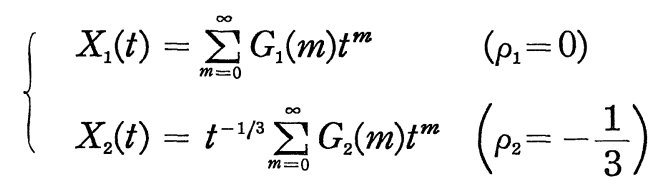

and the formal solutions in the neighbourhood of $t=\infty$ are

$$
\begin{array}{ll}
X^{1}(t) \sim e^{t} t^{-1 / 6} \sum_{s=0}^{\infty} H^{1}(s) t^{-s} & \left(\lambda_{1}=1\right) \\
X^{2}(t) \sim e^{-t} t^{-1 / 6} \sum_{s=0}^{\infty} H^{2}(s) t^{-s} & \left(\lambda_{2}=-1\right) .
\end{array}
$$


The coefficients $G_{1}(m)$ and $G_{2}(m)$ of the convergent power series solutions $X_{1}(t)$ and $X_{2}(t)$ satisfy the following difference equations, together with given initial conditions respectively.

$$
\begin{aligned}
& \left\{\begin{array}{l}
\left(\begin{array}{rr}
m+\frac{1}{6} & -\frac{1}{6} \\
-\frac{1}{6} & m+\frac{1}{6}
\end{array}\right) G_{1}(m)=\left(\begin{array}{rr}
1 & 0 \\
0 & -1
\end{array}\right) G_{1}(m-1) \\
\left(\begin{array}{rr}
\frac{1}{6} & -\frac{1}{6} \\
-\frac{1}{6} & \frac{1}{6}
\end{array}\right) G_{1}(0)=0
\end{array}\right. \\
& \left\{\begin{array}{l}
\left(\begin{array}{rr}
m-\frac{1}{6} & -\frac{1}{6} \\
-\frac{1}{6} & m-\frac{1}{6}
\end{array}\right) G_{2}(m)=\left(\begin{array}{rr}
1 & 0 \\
0 & -1
\end{array}\right) G_{2}(m-1) \\
-\left(\begin{array}{ll}
\frac{1}{6} & \frac{1}{6} \\
\frac{1}{6} & \frac{1}{6}
\end{array}\right) G_{2}(0)=0 .
\end{array}\right.
\end{aligned}
$$

Similarly, the coefficients $H^{1}(s)$ and $H^{2}(s)$ of the formal solutions $X^{1}(t)$ and $X^{2}(t)$ satisfy

$$
\begin{aligned}
& \left\{\begin{array}{l}
\left(\begin{array}{rr}
-s & -\frac{1}{6} \\
-\frac{1}{6} & -s
\end{array}\right) H^{1}(s)=\left(\begin{array}{rr}
0 & 0 \\
0 & -2
\end{array}\right) H^{1}(s+1) \\
\left(\begin{array}{rr}
0 & 0 \\
0 & -2
\end{array}\right) H^{1}(0)=0
\end{array}\right. \\
& \left\{\begin{array}{l}
\left(\begin{array}{cc}
-s & -\frac{1}{6} \\
-\frac{1}{6} & -s
\end{array}\right) H^{2}(s)=\left(\begin{array}{ll}
2 & 0 \\
0 & 0
\end{array}\right) H^{2}(s+1) \\
\left(\begin{array}{ll}
2 & 0 \\
0 & 0
\end{array}\right) H^{2}(0)=0 .
\end{array}\right.
\end{aligned}
$$

According to the result of the previous paper [6], Stokes' multipliers can be determined by solving the linear equations (10) for $m_{0}=0$, since the eigenvalues of the matrix $B$ satisfy the penta- 
gonal condition $1=\left|\lambda_{1}\right|=\left|\lambda_{2}\right|<\left|\lambda_{1}-\lambda_{2}\right|=2$.

In order to solve that linear equations (10) for $m_{0}=0$, we first have to obtain the values of the factorial series

$$
F_{j}^{k}(0)=\sum_{s=0}^{\infty} H^{k}(\mathrm{~s}) g_{j}^{k}(s) \quad(j, k=1,2) .
$$

Since the exact evaluation of this infinite factorial series seems impossible, in general, we will calculate the approximate values of $F_{j}^{k}(0)$.

Now from the difference equations (31), (32), we calculate the coefficients of power series solutions needed later.

$$
\left\{\begin{array}{l}
G_{1}(0)=\left(\begin{array}{l}
1 \\
1
\end{array}\right), \quad G_{1}(1)=\left(\begin{array}{r}
\frac{3}{4} \\
-\frac{3}{4}
\end{array}\right) \\
G_{2}(0)=\left(\begin{array}{r}
1 \\
-1
\end{array}\right), \quad G_{2}(1)=\left(\begin{array}{c}
\frac{3}{2} \\
\frac{3}{2}
\end{array}\right) .
\end{array}\right.
$$

Since the coefficients $g_{j}^{k}(m+s)$ of power series solutions of fundamental equations are given by

$$
g_{j}^{k}(m+s)=\frac{\lambda_{k}^{m+s} \Gamma\left(\rho_{j}-a_{k k}\right)}{\Gamma\left(m+s+\rho_{j}-a_{k k}+1\right)},
$$

we have for $s \geqq 0$,

$$
\left\{\begin{array}{l}
g_{1}^{1}(s)=\frac{\Gamma\left(\frac{1}{6}\right)}{\Gamma\left(s+\frac{7}{6}\right)}=\frac{6^{s+1}}{1 \times 7 \times \cdots \times(6 s+1)} \\
g_{2}^{1}(s)=\frac{\Gamma\left(-\frac{1}{6}\right)}{\Gamma\left(s+\frac{5}{6}\right)}=\frac{6^{s+1}}{(-1) \times 5 \times 11 \times \cdots \times(6 s-1)} \\
g_{1}^{2}(s)=\frac{(-1)^{s} \Gamma\left(\frac{1}{6}\right)}{\Gamma\left(s+\frac{7}{6}\right)}=(-1)^{s} \frac{6^{s+1}}{1 \times 7 \times \cdots \times(6 s+1)}
\end{array}\right.
$$




$$
g_{2}^{2}(s)=\frac{(-1)^{s} \Gamma\left(-\frac{1}{6}\right)}{\Gamma\left(s+\frac{5}{6}\right)}=(-1)^{s+1} \frac{6^{s+1}}{1 \times 5 \times \cdots \times(6 s-1)}
$$

Next, from the estimation formula of the coefficients of formal solutions $H^{k}(s)$, we have

$$
\begin{gathered}
\alpha=\|A\|+\left|a_{k k}\right|=\frac{1}{3}+\frac{1}{6}=\frac{1}{2} \quad(k=1,2), \\
\left\|H^{k}(s+N)\right\| \leqq \frac{\left\|H^{k}(N)\right\|}{2^{s}} \frac{\Gamma\left(N+s+\frac{1}{2}\right)}{\Gamma(N+s)} \quad(k=1,2) .
\end{gathered}
$$

From (37) and (38), we can calculate the remainders of the factorial series (35) which are used as the error estimate as follows,

$$
\begin{aligned}
& \widetilde{F}_{1}^{k}(0)=\left\|\sum_{s=N}^{\infty} H^{k}(s) g_{1}^{k}(s)\right\| \leqq \frac{\| H^{k}(N)\left|\Gamma\left(\frac{1}{6}\right)\right|_{X}}{\Gamma\left(N+\frac{1}{2}\right)} \\
& \sum_{s=0}^{\infty}\left(\frac{1}{2}\right)^{s} \frac{\Gamma\left(s+N+\frac{1}{2}\right)}{\Gamma\left(s+N+\frac{7}{6}\right)} \\
& \widetilde{F}_{2}^{k}(0)=\left\|\sum_{s=N}^{\infty} H^{k}(s) g_{2}^{k}(s)\right\| \leqq \frac{\left.\left|i H^{k}(N) \|\right| \Gamma\left(-\frac{1}{6}\right)\right|_{X}}{\Gamma\left(N+\frac{1}{2}\right)} \times \\
& \sum_{s=0}^{\infty}\left(\frac{1}{2}\right) \frac{\Gamma\left(s+N+\frac{1}{2}\right)}{\Gamma\left(s+N+\frac{5}{6}\right)} \quad(k=1,2) .
\end{aligned}
$$

To obtain the simple estimates of the infinite series in the right hand side of (39), we use Stirling's formula for the gamma function and we have

$$
\frac{\Gamma(z+\alpha)}{\Gamma(z+\beta)}=z^{\alpha-\beta}\left[1+\frac{1}{2} \frac{1}{z}(\alpha-\beta)(\alpha+\beta-1)+O\left(\frac{1}{z^{2}}\right)\right] .
$$

Hence, for a large positive integer $N$, we have 


$$
\begin{aligned}
\frac{\Gamma\left(s+N+\frac{1}{2}\right)}{\Gamma\left(s+N+\frac{7}{6}\right)} & =(s+N)^{-2 / 3}\left[1-\frac{2}{9} \frac{1}{s+N}+O\left(\frac{1}{(s+N)^{2}}\right)\right] \\
& =N^{-2 / 3}\left(\frac{N}{s+N}\right)^{2 / 3}\left[1-\frac{2}{9} \frac{1}{s+N}+O\left(\frac{1}{(s+N)^{2}}\right)\right] \\
& \leqq N^{-2 / 3}
\end{aligned}
$$

Similarly, we have

$$
\frac{\Gamma\left(s+N+\frac{1}{2}\right)}{\Gamma\left(s+N+\frac{5}{6}\right)} \leqq N^{-1 / 3} .
$$

Hence we obtain the following estimates,

$$
\begin{aligned}
& \sum_{s=0}^{\infty}\left(\frac{1}{2}\right)^{s} \frac{\Gamma\left(s+N+\frac{1}{2}\right)}{\Gamma\left(s+N+\frac{7}{6}\right)} \leqq N^{-2 / 3} \sum_{s=0}^{\infty}\left(\frac{1}{2}\right)^{s}=2 N^{-2 / 3} \\
& \sum_{s=0}^{\infty}\left(\frac{1}{2}\right)^{s} \frac{\Gamma\left(s+N+\frac{1}{2}\right)}{\Gamma\left(s+N+\frac{5}{6}\right)} \leqq N^{-1 / 3} \sum_{s=0}^{\infty}\left(\frac{1}{2}\right)^{s}=2 N^{-1 / 3} .
\end{aligned}
$$

Consequently, the estimates of the remainders (39) are

$$
\left\{\begin{array}{l}
\widetilde{F}_{1}^{k}(0) \leqq \frac{\left\|H^{k}(N)\right\|\left|\Gamma\left(\frac{1}{6}\right)\right|}{\Gamma\left(N+\frac{1}{2}\right)} \times 2 N^{-2 / 3} \\
\widetilde{F}_{2}^{k}(0) \leqq \frac{\left\|H^{k}(N)\right\|\left|\Gamma\left(-\frac{1}{6}\right)\right|}{\Gamma\left(N+\frac{1}{2}\right)} \times 2 N^{-1 / 3} \quad(k=1,2) .
\end{array}\right.
$$

Next we shall solve the difference equations (33), (34). We have

$$
H^{1}(0)=\left(\begin{array}{l}
1 \\
0
\end{array}\right), \quad H^{1}(1)=\left(\begin{array}{c}
-\frac{1}{72} \\
\frac{1}{12}
\end{array}\right)
$$

and, if we put

$$
H^{1}(s)=\left(\begin{array}{l}
h_{1}^{1}(s) \\
h_{2}^{1}(s)
\end{array}\right)
$$


we obtain

$$
\left\{\begin{array}{l}
s h_{1}^{1}(s)+\frac{1}{6} h_{2}^{1}(s)=0 \\
2 h_{2}^{1}(s+1)=\frac{1}{6} h_{1}^{1}(s)+s h_{2}^{1}(s)
\end{array}\right.
$$

and, solving the above equations, we have the recurrence formulas as follows,

$$
\left\{\begin{array}{l}
h_{2}^{1}(s+1)=\frac{(6 s-1)(6 s+1)}{72 s} h_{2}^{1}(s) \\
h_{1}^{1}(s+1)=\frac{(6 s-1)(6 s+1)}{72(s+1)} h_{1}^{1}(s) \quad(s \geqq 1) .
\end{array}\right.
$$

Calculating the recurrence formulas (41), we obtain

$$
\left\{\begin{array}{l}
h_{1}^{1}(s)=-\frac{[5 \times 11 \times \cdots \times(6 s-7)][7 \times 13 \times \cdots \times(6 s-5)]}{72^{s} \times s !} \\
h_{2}^{1}(s)=\frac{6 \times[5 \times 11 \times \cdots \times(6 s-7)][7 \times 13 \times \cdots \times(6 s-5)]}{72^{s} \times(s-1) !}
\end{array}\right.
$$

Also we have

$$
H^{2}(0)=\left(\begin{array}{l}
0 \\
1
\end{array}\right), \quad H^{2}(1)=\left(\begin{array}{c}
-\frac{1}{12} \\
\frac{1}{72}
\end{array}\right)
$$

and

$$
\left\{\begin{array}{l}
h_{1}^{2}(s)=(-1)^{s} \times \frac{6 \times[5 \times \cdots \times(6 s-7)][7 \times \cdots \times(6 s-5)]}{72^{s} \times(s-1) !} \\
h_{2}^{2}(s)=(-1)^{s+1} \times \frac{[5 \times \cdots \times(6 s-7)][7 \times \cdots \times(6 s-5)]}{72^{s} \times s !} \quad(s \geqq 2) .
\end{array}\right.
$$

Finally, using the formulas derived above, we can write down the formulas $F_{j}^{k}(0)$ in the form

$$
\begin{aligned}
& F_{1}^{1}(0)=\left\{\begin{array}{ll}
6-\frac{1}{14}+\sum_{s=2}^{N-1} \frac{-6 \times[5 \times \cdots \times(6 s-7)]}{12^{s} \times s ! \times(6 s+1)} & (=\alpha) \\
0+\frac{3}{7}+\sum_{s=2}^{N-1} \frac{36 \times[5 \times \cdots \times(6 s-7)]}{12^{s} \times(s-1) ! \times(6 s+1)} & (=\beta)
\end{array}\right\}+\widetilde{F}_{1}^{1}(0), \\
& F_{2}^{1}(0)=\left\{\begin{array}{ll}
-6+\frac{1}{10}+\sum_{s=2}^{N-1} \frac{6 \times[7 \times \cdots \times(6 s-5)]}{12^{s} \times s ! \times(6 s-1)} & (=\gamma) \\
0-\frac{3}{5}+\sum_{s=2}^{N-1} \frac{-36 \times[7 \times \cdots \times(6 s-5)]}{12^{s} \times(s-1) ! \times(6 s-1)} & (=\delta)
\end{array}\right\}+\widetilde{F}_{2}^{1}(0),
\end{aligned}
$$




$$
\begin{aligned}
& F_{1}^{2}(0)=\left(\begin{array}{l}
\beta \\
\alpha
\end{array}\right)+\widetilde{F}_{1}^{1}(0), \\
& F_{2}^{2}(0)=\left(\begin{array}{l}
\delta \\
\gamma
\end{array}\right)+\widetilde{F}_{2}^{1}(0) .
\end{aligned}
$$

The linear equations

$$
\left\{\begin{array}{l}
G_{1}(0)=\sum_{k=1}^{2} T_{1}^{k} F_{1}^{k}(0), \\
G_{2}(0)=\sum_{k=1}^{2} T_{2}^{k} F_{2}^{k}(0)
\end{array}\right.
$$

are now replaced by approximate equations

$$
\begin{aligned}
& \left(\begin{array}{l}
1 \\
1
\end{array}\right)=T_{1}^{1}\left(\begin{array}{l}
\alpha \\
\beta
\end{array}\right)+T_{1}^{2}\left(\begin{array}{l}
\beta \\
\alpha
\end{array}\right), \\
& \left(\begin{array}{r}
1 \\
-1
\end{array}\right)=T_{2}^{1}\left(\begin{array}{l}
\gamma \\
\delta
\end{array}\right)+T_{2}^{2}\left(\begin{array}{l}
\delta \\
\gamma
\end{array}\right)
\end{aligned}
$$

with solutions

$$
\begin{array}{ll}
T_{1}^{1}=\frac{1}{\alpha+\beta}, & T_{1}^{2}=\frac{1}{\alpha+\beta} \\
T_{2}^{1}=\frac{1}{\gamma-\delta}, & T_{1}^{2}=\frac{-1}{\gamma-\delta} .
\end{array}
$$

Now we shall show an example of the numerical calculation below. Owing to the digital computer and the table of gamma function used, we put $N=6$ and calculate the exact values of seventh order below the point.

The following tables are the results of the numerical computations carried out for $N=6$.

Calculation of $\alpha$

\begin{tabular}{|c|r|r|r|}
\hline$s$ & $-6 \times[5 \times \cdots \times(6 s-7)]$ & $12^{s} \times s ! \times(6 s+1)$ & $\alpha$ \\
\hline 0 & & & $-\frac{1}{14}$ \\
1 & & & -0.0080129 \\
2 & -30 & 3744 & -0.0016752 \\
3 & -330 & 196992 & -0.0004509 \\
4 & -5610 & 12441600 & -0.0001394 \\
5 & -129030 & 92565504 & $\alpha=5.9182930$ \\
\hline
\end{tabular}


Calculation of $\beta$

\begin{tabular}{|c|c|c|c|}
\hline$s$ & $36 \times[5 \times \cdots \times(6 s-7)]$ & $12^{s} \times(s-1) ! \times(6 s+1)$ & $\beta$ \\
\hline 0 & & & 0 \\
1 & & & $\frac{3}{7}$ \\
2 & 180 & 1872 & 0.0961538 \\
3 & 1980 & 65664 & 0.0301535 \\
4 & 33660 & 3110400 & 0.0108218 \\
5 & 774180 & 18513100 & 0.0418180 \\
\hline
\end{tabular}

Calculation of $r$

\begin{tabular}{|c|r|r|r|}
\hline$s$ & $6 \times[7 \times \cdots \times(6 s-5)]$ & $12^{s} \times s ! \times(6 s-1)$ & $r$ \\
\hline 0 & & & -6 \\
1 & & & $\frac{1}{10}$ \\
2 & 42 & 3168 & 0.0132575 \\
3 & 546 & 176256 & 0.0030978 \\
4 & 10374 & 11446272 & 0.0009063 \\
5 & 259350 & 865935360 & 0.0002995 \\
\hline
\end{tabular}

Calculation of $\delta$

\begin{tabular}{|c|r|r|r|}
\hline$s$ & $-36 \times[7 \times \cdots \times(6 s-5)]$ & $12^{s} \times(s-1) ! \times(6 s-1)$ & $\delta$ \\
\hline 0 & & & 0 \\
1 & & & $-\frac{3}{5}$ \\
2 & & & -0.1590909 \\
3 & -252 & 58752 & -0.0557598 \\
4 & -6276 & 2861568 & -0.0217517 \\
5 & -1556100 & 173187072 & -0.0089851 \\
\hline
\end{tabular}

From $\Gamma\left(\frac{1}{6}\right)=\Gamma(0.1 \dot{6})=5.5712058$,

$$
\begin{aligned}
\Gamma\left(\frac{1}{6}\right) & =\Gamma(-0.1 \dot{6})=-6.7776601, \\
\sqrt{\pi} & =1.7724539
\end{aligned}
$$

where in this case the first two values are derived by interpolation from the table [11], the error terms can be calculated as follows, 
(50)

$$
\begin{aligned}
\widetilde{F}_{1}^{1}(0)=\widetilde{F}_{1}{ }_{1}(0)= & \frac{6 \times[5 \times \cdots \times(6 N-7)][7 \times \cdots \times(6 N-5)] \times 2^{N}}{72^{N} \times(N-1) ! \times(2 N-1) \times \cdots \times 3 \times 1 \times \sqrt{\pi}} \\
& \times 2 N^{-2 / 3} \times\left|\Gamma\left(\frac{1}{6}\right)\right| \\
= & 0.0035156
\end{aligned}
$$

$$
\begin{aligned}
\widetilde{F}_{2}^{1}(0)=\widetilde{F}_{2}^{2}(0)= & \frac{6 \times[5 \times \cdots \times(6 N-7)][7 \times \cdots \times(6 N-5)] \times 2^{N}}{72^{N} \times(N-1) ! \times(2 N-1) \times \cdots \times 3 \times 1 \times \sqrt{\pi}} \\
& \times 2 N^{-1 / 3} \times\left|\Gamma\left(-\frac{1}{6}\right)\right| \\
= & 0.0077717 .
\end{aligned}
$$

And from Calculation of $\alpha, \beta, \gamma$ and $\delta$, we have

$$
\begin{aligned}
& \alpha+\beta=6.5258115 \\
& \gamma-\delta=-5.0368514 .
\end{aligned}
$$

Hence, from (48) and (49), we obtain the approximate values of Stokes' multipliers as follows,

$$
\begin{aligned}
& T_{1}^{1}=T_{1}^{2}=\frac{1}{\alpha+\beta}=0.1532376, \\
& T_{2}^{1}=-T_{2}^{2}=\frac{1}{\gamma-\delta}=-0.1985367 .
\end{aligned}
$$

\section{Comparison of the Approximate Values with the Exact Values of Stokes' Multipliers}

Now we shall compare the approximate values derived in section 3 with the exact values derived in section 2 .

If we apply the Theorem 3 of the previous paper [6] to the system of Airy's differential equation (28), we have in the sector $|\arg \bar{t}|<\frac{\pi}{2}$,

$$
\begin{aligned}
X_{1}(\bar{t}) \cong & T_{1}^{1} \Gamma\left(\frac{1}{6}\right) \bar{t}^{-1 / 6} e^{\bar{t}}\left\{\sum_{s=0}^{\sigma} H^{1}(s) \bar{t}^{-s}+O\left(\bar{t}^{-\sigma}\right)\right\} \\
& +T_{1}^{2} \Gamma\left(\frac{1}{6}\right)\left(e^{-\pi i}\right)^{-1 / 6} \bar{t}^{-1 / 6} e^{-\bar{t}}\left\{\sum_{s=0}^{\sigma} H^{2}(s) \bar{t}^{-s}+O\left(\bar{t}^{-\sigma}\right)\right\},
\end{aligned}
$$




$$
\begin{aligned}
X_{2}(\bar{t}) \cong & T_{2}^{1} \Gamma\left(-\frac{1}{6}\right) \bar{t}^{-1 / 6} e^{\bar{t}}\left\{\sum_{s=0}^{\sigma} H^{1}(s) \bar{t}^{-s}+O\left(\bar{t}^{-\sigma}\right)\right\} \\
& +T_{2}^{2} \Gamma\left(-\frac{1}{6}\right)\left(e^{-\pi i}\right)^{1 / 6} \bar{t}^{-1 / 6} e^{-\bar{t}}\left\{\sum_{s=0}^{\sigma} H^{2}(s) \bar{t}^{-s}+O\left(\bar{t}^{-\sigma}\right)\right\}
\end{aligned}
$$

where $\quad \bar{t}=\frac{2}{3} t^{3 / 2}$.

Here the first row of the vector solutions $X_{1}(t)$ corresponds to the solution $x_{0}(t)$ and the first row of the vector solution $X_{2}(t)$ corresponds to the solution $x_{1}(t)$.

Therefore, in order to obtain the exact values of $T_{j}^{k}(j, k=1,2)$, we make the first term coefficient of the first row of $X_{1}(t), X_{2}(t)$ accord with the first term coefficient of $x_{0}(t), x_{1}(t)$ respectively.

From the linear transformation (26), it follows that

$$
\begin{aligned}
P G_{1}(0) & =\left(\begin{array}{rr}
1 & 1 \\
1 & -1
\end{array}\right)\left(\begin{array}{l}
1 \\
1
\end{array}\right)=\left(\begin{array}{l}
2 \\
0
\end{array}\right) \\
P G_{2}(0) & =\left(\begin{array}{rr}
1 & 1 \\
1 & -1
\end{array}\right)\left(\begin{array}{l}
1 \\
-1
\end{array}\right)=\left(\begin{array}{l}
0 \\
2
\end{array}\right) \\
P G_{2}(1) \bar{t}^{2 / 3} & =\left(\begin{array}{rr}
1 & 1 \\
1 & -1
\end{array}\right)\left(\begin{array}{l}
\frac{3}{2} \\
\frac{3}{2}
\end{array}\right) \bar{t}^{2 / 3}=\left(\begin{array}{l}
3 \\
0
\end{array}\right) \bar{t}^{2 / 3}=\left(\begin{array}{l}
3 \\
0
\end{array}\right)\left(\frac{2}{3}\right)^{2 / 3} t .
\end{aligned}
$$

Hence, from (24) and (54), we have the following exact values

$$
\begin{aligned}
& T_{1}^{1} \Gamma\left(\frac{1}{6}\right)\left(\frac{2}{3}\right)^{-1 / 6} \times \frac{1}{2}=\frac{\Gamma\left(\frac{2}{3}\right) 3^{1 / 6}}{2 \sqrt{\pi}}, \\
& T_{2}^{1} \Gamma\left(-\frac{1}{6}\right)\left(\frac{2}{3}\right)^{-1 / 6} \times \frac{1}{3\left(\frac{2}{3}\right)^{2 / 3}}=\frac{\Gamma\left(\frac{1}{3}\right) 3^{-1 / 6}}{2 \sqrt{\pi}},
\end{aligned}
$$

$$
\left\{\begin{array}{l}
T_{1}^{1}=T_{1}^{2}=\frac{\Gamma\left(\frac{2}{3}\right) 2^{1 / 6}}{\Gamma\left(\frac{1}{6}\right) \sqrt{\pi}} \\
T_{2}^{1}=-T_{2}^{2}=\frac{\Gamma\left(\frac{1}{3}\right) 2^{-1 / 6}}{\Gamma\left(-\frac{1}{6}\right) \sqrt{\pi}} .
\end{array}\right.
$$


Here, using the values

$$
\begin{aligned}
& \Gamma\left(\frac{2}{3}\right)=1.3541906 \\
& \Gamma\left(\frac{1}{3}\right)=2.6795252,
\end{aligned}
$$

we calculate numerically the values of the right hand side of (55) and obtain

The results are

$$
\begin{aligned}
& \frac{\Gamma\left(\frac{2}{3}\right) 2^{1 / 6}}{\Gamma\left(\frac{1}{6}\right) \sqrt{\pi}}=0.1539315 \\
& \frac{\Gamma\left(\frac{1}{3}\right) 2^{-1 / 6}}{\Gamma\left(-\frac{1}{6}\right) \sqrt{\pi}}=-0.1987153 .
\end{aligned}
$$

\begin{tabular}{|l|c|c|}
\hline & $T_{1}^{1}=T_{1}^{2}$ & $\varepsilon:$ Estimation error \\
\hline Exact value & 0.1539315 & 0.0035156 \\
Approximate value & 0.1532376 & $\times \quad 2$ \\
\hline Error $(E-A)$ & 0.0006939 & $\varepsilon=0.0070312$ \\
\hline Relative error $\left(\frac{\varepsilon}{E}\right)$ & 0.0045074 & \\
\hline
\end{tabular}

\begin{tabular}{|l|c|c|}
\hline & $T_{2}^{1}=-T_{2}^{2}$ & $\varepsilon:$ Estimation error \\
\hline Exact value & -0.1987153 & 0.0077717 \\
Approximate value & -0.1985367 & $\times \quad 2$ \\
\hline Error $(E-A)$ & -0.0001786 & $\varepsilon=0.0155434$ \\
\hline Relative error $\left(\frac{\varepsilon}{E}\right)$ & -0.0008986 & \\
\hline
\end{tabular}

From the above tables, our method seems to be effective in the numerical determination of Stokes' multipliers even for a general system of the type (1).

\section{Remark on the Determinants of $F_{j}^{k}(m)$ and} the Errors of $T_{j}^{k}$

In the section 1, we explained that Stokes' multipliers of a given system of ordinary differential equations of the type (1) are 
determined by only solving the linear equations

$$
G_{j}(m)=\sum_{k=1}^{n} T_{j}^{k} F_{j}^{k}(m) \quad(j=1,2, \cdots, n)
$$

whenever the factorial series representing $F_{j}^{k}(m)$ are convergent. Hence, it is meaningful to investigate the determinants of the matrix with $n$ column vectors $F_{j}^{k}(m)$ in more detail apart from the approximate evaluations of $F_{j}^{k}(\imath n)$.

If we consider $F_{j}^{k}(m)$ as functions in $m, F_{j}^{k}(m)$ are indeed the general solutions of the difference equations satisfied by the coefficients $G_{j}(m)$ of convergent power series solutions, that is, $F_{j}^{k}(m)$ satisfy the following difference equations

$$
\left(\rho_{j}+m-A\right) F_{j}^{k}(m)=B F_{j}^{k}(m-1) .
$$

Now, we denote the determinants of $F_{j}^{k}(m)$ by $C_{j}(m)$ as follows,

$$
C_{j}(m)=\operatorname{det}\left\{F_{j}^{1}(m), F_{j}^{2}(m), \cdots, F_{j}^{n}(m)\right\} .
$$

From the difference equations (57), we have

$$
\prod_{k=1}^{n}\left(\rho_{j}+m-\rho_{k}\right) \cdot C_{j}(m)=\prod_{k=1}^{n} \lambda_{k} \cdot C_{j}(m-1) .
$$

Therefrom, we obtain

$$
C_{j}(m)=\prod_{k=1}^{m} \frac{\lambda_{k}^{m} \Gamma\left(\rho_{j}-\rho_{k}+1\right)}{\Gamma\left(\rho_{j}+m-\rho_{k}+1\right)} C_{j}(0) .
$$

On the other hand, for a large positive integer $m$, we have the following asymptotic forms from the factorial series expansion,

$$
F_{j}^{k}(m)=H^{k}(0) g_{j}^{k}(m)\left\{1+O\left(\frac{1}{m}\right)\right\} .
$$

Since the eigenvectors $H^{k}(0)(k=1,2, \cdots, n)$ are linearly independent and the $\operatorname{det}\left\{H^{1}(0), H^{2}(0), \cdots, H^{n}(0)\right\}=1$, we have

$$
\begin{aligned}
C_{j}(m) & =\left(\prod_{k=1}^{n} g_{j}^{k}(m)\right)\left\{1+O\left(\frac{1}{m}\right)\right\} \\
& =\prod_{k=1}^{n} \frac{\lambda_{k}^{m} \Gamma\left(\rho_{j}-a_{k k}\right)}{\Gamma\left(m+\rho_{j}-a_{k k}+1\right)} 1+O\left(\frac{1}{m}\right\} .
\end{aligned}
$$


Hence, from (59) and (60), we obtain

$$
C_{j}(0)=\left(\prod_{k=1}^{n} \frac{\Gamma\left(m+\rho_{j}-\rho_{k}+1\right)}{\Gamma\left(m+\rho_{j}-a_{k k}+1\right)} \frac{\Gamma\left(\rho_{j}-a_{j k}\right)}{\Gamma\left(\rho_{j}-\rho_{k}+1\right)}\right)\left\{1+O\left(\frac{1}{m}\right)\right\} .
$$

Here, if $m$ tends to infinity, by Stirling's formula

$$
\frac{\Gamma\left(m+\rho_{j}-\rho_{k}+1\right)}{\Gamma\left(m+\rho_{j}-a_{k k}+1\right)}=m^{a_{k k}-\rho_{j}}\left\{1+O\left(\frac{1}{m}\right)\right\}
$$

and by the invariance of the trace of a matrix

$$
\sum_{k=1}^{n} a_{k k}=\sum_{k=1}^{n} \rho_{k},
$$

we obtain

$$
C_{j}(0)=\prod_{k=1}^{n} \frac{\Gamma\left(\rho_{j}-a_{k k}\right)}{\Gamma\left(\rho_{j}-\rho_{k}+1\right)} .
$$

But this is an identity relation because $C_{j}(0)$ is independent of $m$. Finally, we obtain

$$
C_{j}(m)=\prod_{k=1}^{n} \frac{\lambda_{k}^{m} \Gamma\left(\rho_{j}-a_{k k}\right)}{\Gamma\left(\rho_{j}+m-\rho_{k}+1\right)}
$$

which we can calculate explicitly independent of the values of $F_{j}^{b}(m)$.

Now we shall solve the linear equation (56) for a certain appropriate positive integer $m_{0}$. The exact values of Stokes' multipliers are given by

$$
T_{j}^{k}=\frac{\operatorname{det}\left\{F_{j}^{1}\left(m_{0}\right) \cdot F_{j}^{2}\left(m_{0}\right) \cdots \stackrel{h}{G}_{j}^{k}\left(m_{0}\right) \cdots F_{j}^{n}\left(m_{0}\right)\right\}}{C_{j}\left(m_{0}\right)} \quad(j, k=1,2, \cdots, n) .
$$

But, as described in the preceding sections, we shall not, in general, be able to calculate the exact values of $F_{j}^{k}\left(m_{0}\right)$.

So, we substitute the sums of the first arbitrary $N$ terms $F_{j N}^{k}\left(m_{0}\right)$ for $F_{j}^{k}\left(m_{0}\right)$ and obtain the approximate values of Stokes' multipliers as follows.

$$
\hat{T}_{j}^{k}=\frac{\operatorname{det}\left\{F_{j_{N}}^{1}\left(m_{0}\right), F_{j N}^{2}\left(m_{0}\right) \cdots \stackrel{b}{k}\left(m_{0}\right) \cdots F_{j}^{n} N\left(m_{0}\right)\right\}}{C_{j}\left(m_{0}\right)} .
$$

Then, using the norm estimates, the errors $\varepsilon_{j}^{k}$ are 


$$
\varepsilon_{j}^{k}=\frac{\operatorname{det}\left\{\widetilde{F}_{j}^{1}\left(m_{0}\right), \widetilde{F}_{j}^{2}\left(m_{0}\right) \cdots \stackrel{v}{G}_{j}\left(m_{0}\right) \cdots \widetilde{F}_{j}^{n}\left(m_{0}\right)\right\}}{C_{j}\left(m_{0}\right)}
$$

From (66), we may say that the smaller the absolute values of $C_{j}\left(m_{0}\right)$ will be, the greater the errors will become. But $C_{j}\left(m_{0}\right)$ never vanish, because, in that case, $\rho_{j}-\rho_{k}=-m_{1}\left(m_{1} \geqq m_{0}+1\right)$ is satisfied for some $k$ and we have convergent power series solutions involving logarithmic functions and the different linear equations determining Stokes' multipliers from (56). See [4], [5].

In conclusion, our method is effective except for the case when it is difficult to judge the appearance of the logarithmic terms.

\section{References}

[1] Birkhoff, G. D., Collecied mathematical papers, Amer. Math. Soc., New York, 1950.

[2] Knobloch, H. W., Zusammenhänge zwischen konvergenten und asymptotischen Entwicklungen bei Lösungen linearer Differentialsystems von Range Eins, Math. Ann. 134 (1958), 206-288.

[3] Okubo, K., A global representation of a fundamental set of solutions and a Stokes phenomenon for a system of linear differential equations, J. Math. Soc. Japan, 15 (1963), 268-288.

[4] - A connection problem involving a logarithmic function, Publ. RIMS Kyoto Univ. Ser. A, 1 (1965), 99-128.

[5] Kohno, M., A two points connection problem involving logarithmic polynomials, Ibid. 2 (1966), 269-305.

[6] - The convergence condition of a series appearing in connection problems and the determination of Stokes' multipliers, Ibid. 3 (1968), 337-350.

[7] Erdélyi, A., Asymptotic expansions, Dover, New York, 1956.

[8] Friedrichs, K. O., Special topics in analysis, Lecture note, New York University, 1953-1954.

[9] Wasow, W., Asymptotic expansions for ordinary differential equations, Interscience, 1966.

[10] Abramowitz, M. and I. A. Stegun, Handbook of mathematical functions, Dover, New York.

[11] Davis, H. T., Tables of the mathematical functions, vol. 1, Trinity University, 1963

\section{Eratta}

In the Theorem 3 of the previous paper "The convergence condition of a series appearing in connection problems and the 
determination of Stokes' multipliers", the author commited a small error of dropping the constants $\Gamma\left(\rho_{j}-a_{k k}\right)$ which correspond to $C_{*}^{k}$ in the Theorem. 5.3. of the paper "A two points connection problem involving logarithmic polynomials".

Since in the former paper a two point connection problem involving no logarithmic terms is treated, the constants $C_{*}^{k}$ involve no values of the derivatives of gamma function as the case of the latter paper and are easily given by $c_{0}=\lambda_{k}^{a_{k k}-\rho_{j}} \Gamma\left(\rho_{j}-a_{k k}\right)$. (see the latter paper, 277 p., 304 p.).

Therefore the constant $\Gamma\left(\rho_{j}-a_{k k}\right)$ should be attached between $\lambda_{k}^{a_{k k^{-}}-\rho_{j}}$ and $e^{\lambda_{k} t}$ in the formulas of the following rows from $348 \mathrm{p}$. to $350 \mathrm{p}$.

\begin{tabular}{|c|c|c|}
\hline $348 p$ & $349 \mathrm{p}$ & $350 \mathrm{p}$ \\
\hline 11 th row & 7 th row & 3 th row \\
\hline 15 th row & 10 th row & 11 th row \\
\hline 16 th row & 22 th row & 14 th row \\
\hline 24 th row & \\
\hline
\end{tabular}

The correct connection formula follows

$$
X_{j}(t) \cong \sum_{k=1}^{n} T_{j}^{k} \lambda_{k}^{a_{k k}-\rho_{j}} \Gamma\left(\rho_{j}-a_{k k}\right) X^{k}(t)
$$

